\title{
О динамике обратимой сорбции антоцианов на поверхности бентонитовых глин
}

\author{
(C) 2020 Дейнека В.И., Доронин А.Г., Скрыпников Н.С., Дейнека Л.А. \\ ФГАОУ ВО «Белгородский государственный наџиональный исследовательский университет», \\ Белгород
}

Поступила в редакцию 30.08.2020 г.

DOI: $10.17308 /$ sorpchrom.2020.20/3050

На примере сорбции антоцианов плодов паслена черного садового на бентонитовой глине «Бентакон» показано, что при применении уравнения Лагергрена необходимо из набора экспериментальных данных исключить результаты, полученные в начале и в конце эксперимента. Уравнение Лагергрена в модифицированном для использования прямых результатов измерений связано с временем $\tau$ от начала сорбции линейным уравнением $\lg \left(A_{\tau}-A_{\mathrm{e}}\right)=a_{0}-a_{1} \tau$, где $A_{\tau}$ и $A_{e}-$ оптическая плотность растворов антоцианов в растворах во время $\tau$ и при окончании сорбции. Ранее предложенное исправленное уравнение динамики необратимой сорбции на сорбентах без внутридиффузионного торможения, учитывающее изменения концентрации сорбата в растворе при сорбции, позволяет включить в набор данных для обработки все начальные точки, однако при этом данные в конце сорбционной кривой располагаются выше полученной линии тренда. Высказано предположение, что данный факт связан с обратимостью сорбции антоцианов в использованных при проведении эксперимента условиях. Для численного моделирования обратимой сорбции использовали пакет MS Excel. B работе изложен алгоритм моделирования, в результате применения которого показано, что и при обратимости сорбции линейность по исправленному уравнению для необратимой сорбции сохраняется до достижения 70$80 \%$-ного завершения сорбции, что устанавливает границы применимости исправленного уравнения: $\mathrm{R}^{2}$ для линейной зависимости не ниже 0.99 .

Ключевые слова: уравнения динамики сорбции, непористые сорбенты, антоцианы, бентонитовые глины, численное моделирование, обратимая сорбция, MS Excel.

\section{Введение}

При анализе динамики необратимой сорбции веществ из жидкой фазы на твердых сорбентах без внутридиффузионного (т.е. в порах сорбента) торможения широко используется уравнение Лагергрена [1]. Уравнение выводится в условиях приближения сорбции необратимой реакцией псевдо-первого порядка, не учитывающей изменение концентрации сорбируемых веществ в жидкой фазе:

$$
\frac{d Q_{\tau}}{d \tau}=k_{1}\left(Q_{e}-Q_{\tau}\right)
$$

где $\tau$ - время с начала сорбции; $Q_{\tau}-$ степень (лучше - уровень) заполнения поверхности сорбента в момент времени $\tau$; $k_{1}$ - константа скорости сорбции; $Q_{e}$ - уровень заполнения поверхности при насыщении. Интегрированием уравнения (1) получают более удобное для обработки уравнение (2):

$$
\lg \left(Q_{e}-Q_{\tau}\right)=\lg \left(Q_{e}\right)-\frac{k_{1}}{2,303} \tau .
$$

В качестве потенциальных систем для обработки по данному уравнению подходит сорбция антоцианов на бентонитовых глинах, содержащих в качестве важней- 
шего минерала монтмориллонит [2]. Ионообменные свойства минерала связаны с гетеровалентным замещением ионов $\mathrm{Al}^{3+}$ (обычно) на ионы $\mathrm{Mg}^{2+}$ в октаэдрическом металл-гидроксидном слое [2], закрытом с обеих плоскостей тетраэдрическими кремний-кислородным слоями, благодаря чему такой суммарный слой приобретает отрицательный заряд. Отрицательный заряд в монтмориллоните компенсируется противоионами в межслоевом пространстве, которые и обеспечивают ему способность к набуханию. Важной специфической особенностью такого минерала является отсутствие на поверхности отрицательно заряженных функциональных групп - заряд делокализован между несколькими (шестью) атомами кислорода. Это обеспечивает энергетическую однородность центров ионообменной сорбции удовлетворяя условиям, налагаемым на сорбцию по Ленгмюру, подтвержденную ранее выполненными исследованиями по сорбции антоцианов на бентонитовых глинах [3].

Поскольку бентонитовые глины в водной смеси могут существовать в виде отделенных друг от друга сольватированных водой слоев при отсутствии каких-либо поровых пространств, то диффузионное торможение в порах отсутствует. Именно эта способность бентонитовых глин лежит в основе обычного способа обогащения природных осадочных пород седиментацией, при которой частицы сопутствующих минералов осаждаются быстрее монтмориллонита.

Уравнение (2) удобнее переписать для использования реально измеряемых концентраций или оптических плотностей сорбата в растворе с учетом того, что:

$$
\begin{aligned}
& Q_{e}=k_{1}\left(c_{0}-c_{e}\right)=k_{2}\left(A_{0}-A_{e}\right) \\
& Q_{\tau}=k_{1}\left(c_{0}-c_{\tau}\right)=k_{2}\left(A_{0}-A_{\tau}\right) \\
& \quad \lg \left(A_{\tau}-A_{e}\right)=\lg \left(A_{0}-A_{e}\right)-\frac{k_{1}}{2,303} \tau .
\end{aligned}
$$

где $A_{e}, A_{\tau}$ и $A_{0}$ - оптическая плотность растворов антоцианов в растворах при окончании сорбции, во время $\tau$ от начала сорбции и в начале эксперимента, соответственно; $k_{1}$ и $k_{2}-$ коэффициенты для перехода от разности концентраций или оптических плотностей растворов к степени заполнения.

Уравнения (2) и (3) можно применять к результатам эксперимента и при отличии процесса от первого порядка, но параметр $\left(Q_{e}-Q_{\tau}\right)$ при этом обычно не соответствует числу свободных центров, как и параметр $Q_{e}$ обычно не равен отрезку, отсекаемому прямой линией на графике по уравнениям (2) или (3) [4]. Одной из причин этих несоответствий может быть не учёт изменения концентрации сорбата в маточном растворе в процессе сорбции. Но эта проблема легко устранима использованием другого улучшенного уравнения [5], в котором исходное уравнение (1) заменено на уравнение, учитывающее концентрацию сорбата в растворе:

$$
-\frac{d c_{\tau}}{d \tau}=k_{1} c_{\tau} \theta_{\text {св. } \tau}
$$

где $c_{\tau}$ - концентрация вещества в фазе раствора; $\tau$ - время с начала сорбции; $k_{1}-$ константа скорости сорбции по псевдо-первому порядку; $\theta_{c в . \tau}-$ количество свободных мест на поверхности сорбента. Данное уравнение имеет решение в аналитическом виде [6]:

$$
\ln \left(\frac{c_{\tau}}{c_{\tau}-c_{e}}\right)=\ln \left(\frac{A_{\tau}}{A_{\tau}-A_{e}}\right)=c_{e} k_{1} k_{2} \tau+\ln \left(\frac{c_{0}}{c_{0}-c_{e}}\right)=a \tau+b,
$$

где $k_{2}$ - константа, связывающая изменение концентрации вещества в растворе с изменением числа мест на поверхности сорбента, начальную и конечные концентрации вещества в растворе; $\tau$ - время с начала сорбции; $c_{0}$ и $c_{\mathrm{e}}-$ начальная и конечная концентрации сорбата в растворе.

Однако, исследования [6] показывают, что сорбция антоцианов на бентонитовых глинах реально обратима. Поэтому необходима оценка влияния обратимости 
сорбции на результаты, получаемые при анализе экспериментальных данных по исправленному уравнению (5), что и стало целью настоящего исследования.

\section{Экспериментальная часть}

Антоцианы получали экстракцией $0.1 \mathrm{M}$ водным раствором $\mathrm{HCl}$ из растительных материалов. Полученные экстракты очищали сорбцией на бентонитовой глине, с которой антоцианы десорбировали смесью $0.1 \mathrm{M}$ водный раствор $\mathrm{HCl}$ - этанол в соотношении 1:1. Этанол отгоняли на вакуумном ротационном испарителе и остающийся раствор разбавляли $0.1 \mathrm{M}$ водным раствором $\mathrm{HCl}$ для исследования динамики сорбции. В работе использовали бентонитовую глину Бентокон «Медиум» (ООО «Бентопром»)

При исследовании динамики сорбции к подготовленному раствору антоцианов в $0.1 \mathrm{M}$ водном растворе $\mathrm{HCl}$ объемом $150 \mathrm{~cm}^{3}$ добавляли навеску бентонитовой глины (0.030-0.100 г) и включали перемешивание. Через каждые 5 мин отбирали аликвотные объемы смеси $\left(5 \mathrm{~cm}^{3}\right)$, центрифугировали 3 мин, и определяли оптическую плотность раствора. Раствор с осадком возвращали в исходную смесь.

Концентрацию антоцианов определяли прямым спектрофотометрическим методом в кварцевых кюветах на приборе Shimadzu UV-1550 при pH=1 без дифференциального подхода [7], поскольку полимерные антоцианы отделялись на стадии очистки.

\section{Обсуждение результатов}

При сорбции антоцианов паслена садового при комнатной температуре была исследована динамика сорбции. Полученные результаты, рис.1, свидетельствуют о том, что сорбционное равновесие наступает примерно за 0.5 ч, что говорит об отсутствии внутридиффузионных торможений, т.е. в водной смеси пакеты глины не образуют агрегатов с пористой структурой (которую обнаруживают при низкотемпературной сорбции азота на образцах сухой глины). При этом сорбционная емкость бентонитовой глины оказалась не очень высокой - менее 25 мкмоль/г.

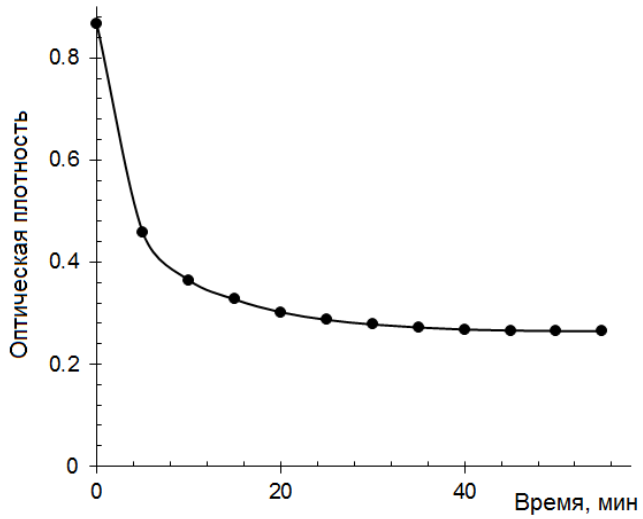

Рис. 1 Изменение оптической плотности экстракта плодов паслена садового при сорбции на бентонитовой глине «Бентокон»

Fig. 1. Changes in the optical density of the nightshade fruit extract during sorption on bentonite clay «Bentokon»

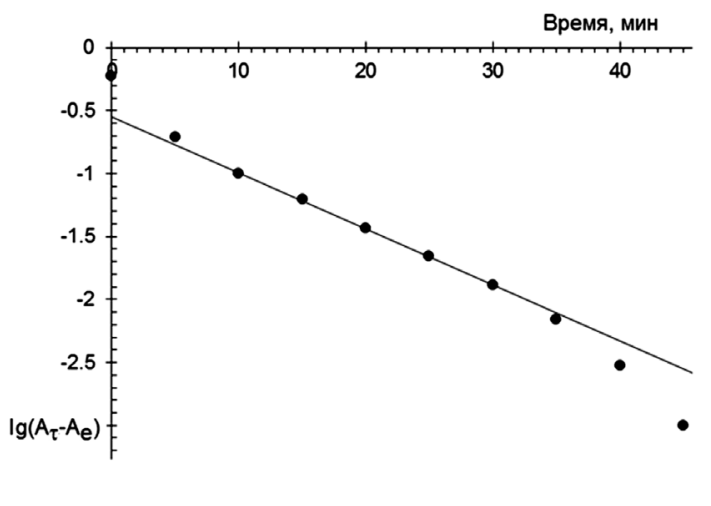

Рис. 2 Анализ динамики сорбции антоцианов плодов паслена по уравнению Лагергрена (уравнение 2)

Fig. 2. Analysis of the dynamics of sorption of anthocyanins from nightshade fruits according to Lagergren's equation (equation 2) 
При обработке полученных данных по уравнению Лагергрена, рис. 2, первые две экспериментальные точки, как обычно [4], существенно отклонялись (вверх) от прямой линии, проведенной по точкам для интервала времен сорбции $(10,30)$ мин. При этом точки для более длительного времени (для более полной сорбции) располагаются ниже этой прямой линии.

Обработка тех же данных в координатах улучшенного уравнения (4), рис. 3, позволяет захватить диапазон экспериментальных данных в диапазоне времен от 0 до 30 мин. Отклонение от полученной прямой точек для больших времен сорбции может быть следствием обратимости процесса сорбции антоцианов на бентонитовых глинах.

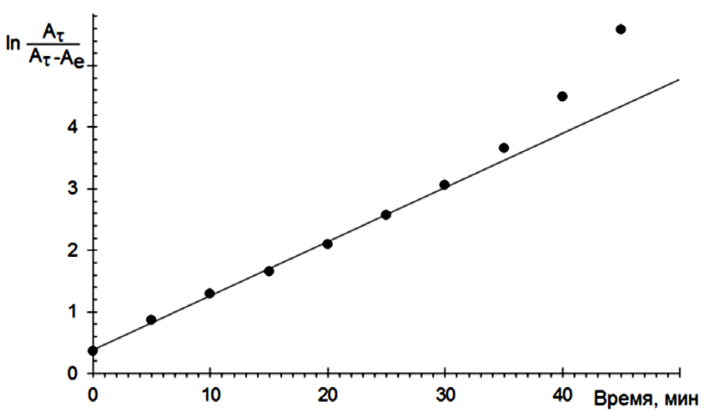

Рис. 3 Анализ динамики сорбции антоцианов плодов паслена по исправленному уравнению (4)

Fig. 3. Analysis of the dynamics of sorption of anthocyanins from nightshade fruits according to the corrected equation (4)

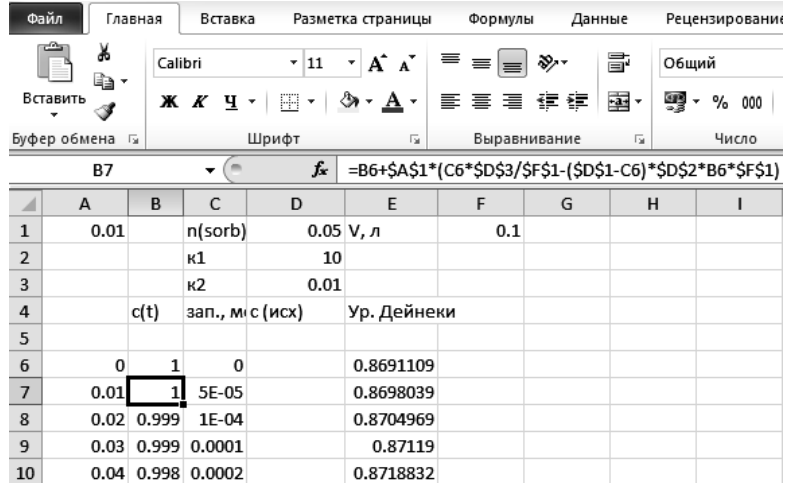

Рис. 4. Фрагмент рабочего листа Ехcel для численного моделирования динамики обратимой сорбции

Fig. 4. Fragment of an Excel worksheet for numerical modelling of the dynamics of reversible sorption

Для проверки применимости и оценки диапазона применимости исправленного уравнения (4) для обратимой сорбции было выполнено компьютерное моделирование сорбции в программе Excel. В методе использовали уравнение для обратимой сорбции:

$$
-\frac{d c_{\tau}}{d \tau}=k_{1} c_{\tau} \theta_{\text {св. } \tau}-k_{2} \theta_{\text {зан. } \tau},
$$

где $k_{1}$ и $k_{2}-$ константы скоростей сорбции и десорбции, соответственно; $c_{\tau}-$ концентрация антоцианов в растворе в момент времени $\tau, \theta_{\text {св. } \tau}$ и $\theta_{3 а н . \tau}-$ доли свободных и занятых мест на поверхности сорбента, соответственно.

Воспользуемся возможностями программы Excel для численного расчета кривых динамики сорбции при условии обратимой сорбции. Для этого составим рабочий лист в программе Excel, фрагмент которого представлен на рис. 4.

В этом рабочем листе задается время (столбец А), $\tau$, начиная с 0 (ячейка А6) с шагом, указанным в ячейке А1. В ячейке В6 помещается начальная концентрация антоцианов в растворе (в условных единицах, пропорциональных числу моль/дм ${ }^{3}$ ). В ячейке записана D1 - предельная емкость сорбента по антоцианам, а в ячейках D2 и D3 - константы скоростей сорбции и десорбции, соответственно, и, наконец, в ячейке F1 - объем экстракта в дм³. Теперь в ячейки В7 и С7 вводим формулы для расчета соответствующих величин:

- для ячейки В7: =B6+\$A \$1*(C6*\$D\$3/\$F\$1-(\$D\$1-C6)*\$D\$2*B6*\$F\$1),

- для ячейки $\mathrm{C} 7=\mathrm{C} 6+(\mathrm{B} 6-\mathrm{B} 7) * \$ \mathrm{~F} \$$. 
Это позволяет сформировать столбцы данных на любое время с любыми варьируемыми параметрами. При этом столбец Е используем для расчета натурального логарифма соотношения $c_{\tau}$ к $\left(c_{\tau}-c_{e}\right)$ левой части уравнения (4). Результаты, полученные для некоторых исходных параметров, представлены на рис. 5.

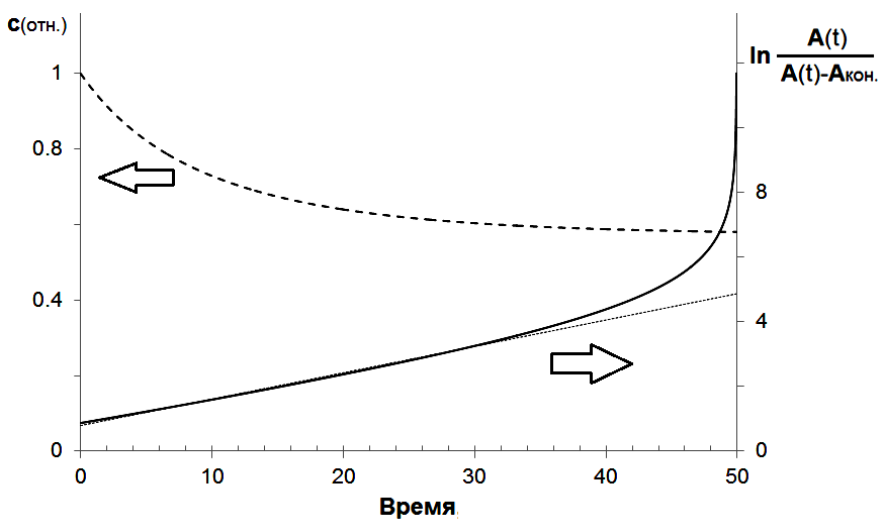

Рис. 5 Динамика обратимой сорбции антоцианов на бентонитовой глине

Fig. 5. Dynamics of reversible sorption of anthocyanins on bentonite clay

Из представленных данных следует, что экспериментальные данные удовлетворительно описываются прямолинейной зависимостью функции от концентрации антоцианов в маточном растворе от времени по уравнению (4) примерно до 70-80\%ной степени насыщения сорбента, а в дальнейшем экспериментальные данные должны вследствие обратимости процесса располагаться выше этой линии тренда.

Для количественной оценки возможности применимости исправленного уравнения необратимой сорбции для результатов равновесной сорбции, был выполнен расчет параметров уравнения (4), записанном в виде уравнения (5), при различных вариантах ограничения числа начальных точек, использованных при построении прямой линии:

$$
\ln \frac{A_{\tau}}{A_{\tau}-A_{e}}=a_{0}+a_{1} \cdot t .
$$

Ограничение числа используемых при построении прямой линии точек (из полного набора данных) проводили по достижению соответствующей степени заполнения поверхности сорбента (степень сорбции, $\alpha$ ), табл. 1 в условиях, при которых изменение концентрации антоцианов при достижении равновесия составило около $42 \%$, табл. 1.

Таблица 1 Параметры уравнения необратимой сорбции при ограничении используемых точек по степени сорбции

Table 1. Parameters of the equation of irreversible sorption when limiting the points used by the degree of sorption

\begin{tabular}{|c|c|c|c|}
\hline \multirow{2}{*}{ Степень сорбции, $\alpha, \%$} & \multicolumn{3}{|c|}{ Параметры уравнения (5) } \\
\cline { 2 - 4 } & $a_{0}$ & $a_{1}$ & $\mathrm{R}^{2}$ \\
\hline 40.3 & 0.850 & 0.075 & 0.9996 \\
\hline 50.3 & 0.837 & 0.077 & 0.9992 \\
\hline 60.4 & 0.818 & 0.079 & 0.9986 \\
\hline 70.5 & 0.791 & 0.082 & 0.9974 \\
\hline 80.6 & 0.745 & 0.085 & 0.9945 \\
\hline 90.7 & 0.667 & 0.091 & 0.6863 \\
\hline
\end{tabular}


Параметр $\mathrm{R}^{2}$ был не ниже 0.99 вплоть до 80\%-ной степени насыщения поверхности. При этом и параметр $a_{1}$, который, в отличие от параметров уравнения Лагергрена, сохраняет физический смысл и может быть использован для расчета константы скорости сорбции антоцианов, может быть определен с погрешностью, не превышающей $10 \%$ при использовании точек до $70 \%$-ного уровня насыщения поверхности сорбента, и меньше 5\% при включении в набор экспериментальных точек до 60\%ного насыщения.

\title{
Заключение
}

Таким образом, исправленное уравнение для необратимой сорбции антоцианов на поверхности не содержащих пор сорбентов (в отличие от уравнения Лагергрена), учитывающее изменение концентрации сорбата в растворе при сорбции, может быть применимо для обработки экспериментальных данных вплоть до достижения 80\%ной степени заполнения поверхности сорбента даже при обратимости процесса сорбции-десорбции антоцианов. В любом случае, возможна оценка применимости любых уравнений по методу моделирования сорбции с использованием программы MS Excel.

\section{Список литературы}

1. Ho Y.-S. // Scientometrics. 2004. Vol. 59. pp. 171-177.

2. Park J.-H., Shin H.-J., Kim M.H., Kim J.-S. et al. // J. Pharm. Investigation. 2016. Vol. 46. pp. 363-375.

3. Чулков А.Н., Дейнека В.И., Тихова А.А., Везенцев А.И. и др. // Ж. физ. химии. 2012. Т. 86. С. 500-502.

4. Крижановская О.О., Синяева Л.А., Карпов С.И., Селеменев В.Ф. и др. // Сорбиионные и хроматографические прочессы. 2014. T. 14. № 5. C. 784-794.
5. Дейнека В.И., Доронин А.Г., Дейнека Л.А. // Ж. физ. химии. 2020. Т. 94. С. 121-124

6. Дейнека В.И., Доронин А.Г., Олейниц Е.Ю., Блинова И.П. и др. // Ж. физ. химии. 2020. T. 94. С. 920-925.

7. Дейнека Л.А., Сидоров А.Н., Дейнека В.И., Кульченко Я.Ю. и др.// Ж. аналит. химии. 2020. Т. 75. С. 510-515.

\section{Dynamics of reversible sorption of anthocyanins on the surface of bentonite clays}

\author{
(C) 2020 Deineka V.I., Doronin A.G., Skrypnikov N.S., Deineka L.A. \\ Belgorod State National Research University, Belgorod, Russian Federation
}

\begin{abstract}
Using examples of sorption of anthocyanins of black garden nightshade (known as the Sunberry) fruits on bentonite clay «Bentakon», it was shown that when applying the Lagergren's equation, it is necessary to exclude the results obtained at the beginning and at the end of the experiment from the set of experimental data. Lagergren's equation modified for the use of direct measurements is associated with the time $\tau$ from the beginning of the sorption by the linear equation $\lg \left(A_{\tau}-A_{\mathrm{e}}\right)=a_{0}-a_{1} \tau$, where $A_{\tau}$ and $A_{e}$ are the absorbance of anthocyanins in solutions at the time $\tau$ and at the end of the sorption. The previously proposed correction to the equation for the dynamics of irreversible sorption on sorbents without intradiffusion inhibition, taking into account changes in the concentration of sorbate in the solution during the sorption of $\ln (\mathrm{A} \tau /(\mathrm{A} \tau-\mathrm{Ae}))=\mathrm{a} 0+$ a1 $\tau$ allows the inclusion of all the starting points into the data set processed by the equation, the data at the end of the sorption curve were located above the obtained trend line. It was suggested that this was due to the reversibility of the sorption of anthocyanins under experimental conditions. MS Excel was used for the numerical simulation of reversible sorption. The study presents a simulation algorithm, which shows that even in the case of the reversibility of sorption, the linearity of the corrected equation for irreversible sorption is preserved
\end{abstract}


until the $70-80 \%$ completion of sorption is reached, which sets the limits of applicability of the corrected equation: R2 for a linear dependence was at least 0.99 .

Keywords: equations of sorption dynamics, nonporous sorbents, anthocyanins, bentonite clays, numerical modelling, reversible sorption, MS Excel.

\section{References}

1. Ho Y.-S., Scientometrics, 2004, Vol. 59, pp. $171-177$.

https://doi.org/10.1023/B:SCIE.0000013305.99 473.cf.

2. Park J.-H., Shin H.-J., Kim M.H. et al., J. Pharm. Investigation, 2016, Vol. 46, pp. 363375. DOI 10.1007/s40005-016-0258-8.

3. Chulkov A.N., Deineka V.I., Tikhova A.A. et al., Russ. J. Phys. Chem. A, 2012, Vol. 86, pp. 429-431.

https://doi.org/10.1134/S0036024412030089.

4. Krizhanovskaya O.O., Siniaeva L.A., Karpov S.I. et al., Sorbtsionnye i khromatograficheskie protsessy, 2014, Vol. 14, No 5, pp. 784-794.

Дейнека Виктор Иванович - профессор кафедры общей химии, д.х.н., Белгородский государственный национальный исследовательский университет, Белгород

Доронин Андрей Геннадьевич - аспирант кафедры общей химии, д.х.н., Белгородский государственный национальный исследовательский университет, Белгород

Скрыпников Николай Сергеевич - студент Белгородского государственного национального исследовательского университета, Белгород

Дейнека Людмила Александровна - доцент кафедры общей химии, к.х.н., Белгородский государственный национальный исследовательский университет, Белгород
5. Deineka V.I., Doronin A.G., Deineka L.A., Russian Journal of Physical Chemistry A, 2020, Vol. 94, No 1, pp. 167-170. DOI: 10.1134/S0036024420010057.

6. Deineka V.I., Doronin A.G., Oleinits E.Yu. et al., Russian Journal of Physical Chemistry A, 2020, Vol. 94, No 6, pp. 1224-1229. DOI: $10.1134 / \mathrm{S} 0036024420060072$.

7. Deineka L.A., Sidorov A.N., Deineka V.I. et al., Journal of Analytical Chemistry, 2020, Vol. 75, No 6, pp. 754-758. DOI: $10.1134 / \mathrm{S} 1061934820060064$.

Deineka Viktor, Professor of the Department of General Chemistry Belgorod state national research University, Belgorod, Belgorod, e-mail deineka@bsu.edu.ru

DoroninAndrey - post-graduate of the Department of General Chemistry Belgorod state national research University, Belgorod, Belgorod, e-mail 634212@bsu.tdu.ru

Skrypnikova Nikolay S. - student of Belgorod state national research University, Belgorod, Belgorod, e-mail 812887@bsu.edu.ru

Deineka Lyudmila A. - Associate Professor of the Department of General Chemistry Belgorod state national research University, Belgorod, e-mail deyneka@bsu.edu.ru 